Richard Maly: Ueber d. Verwirrungen u. Entstellungen i. d. Peptonlehre. 315

\title{
Ueber die Verwirrungen und Entstellungen in der Peptonlehre.
}

Von

\section{Richard Maly}

in Graz.

Bei weitem nicht die einzige aber die letzte und unmittelbarste Ursache, für das was ich hier vorzubringen habe, ist eine jüngst aus dem Laboratorium Hoppe-Seyler's datirte Notiz eines Herrn Kossel ${ }^{1}$ ): „über die ehemische Zusammensetzung der Peptone", in der versucht wird, gewisse ältere klägliche Resultate, auch jetzt, nachdem bessere Finsicht von anderer Seite längst gefördert worden ist, doch noch zu vertheidigen, selbst auf Kosten der offenbar schon erkannten aber nicht zugestandenen Wahrheit.

Solchen Eindruck macht die genannte Mittheilung, die von Seite des Autors am besten unterdrückt worden wäre, denn für jene, welche die Entwicklung der Peptonlehre kennen, und sich ihr nicht absichtlich zu verschliessen Anlass haben, ist die empirische Zusammensetzung des Peptons und seine Natur als unfällbares Eiweiss ebenso wenig ein Punkt der Discussion mehr, als die damit im innigsten Zusammenhange stehende physiologische Bedeutung desselben. Der Versuch des Hr. Kossel war daher irrelevant, von einer s o verlornen Sache ist auch zum Scheine nichts zu retten. Wenn ich trotzdem an eine der Entgegnung nicht werthe Notiz hier anknüpfe, so geschieht dies nur, weil ich dahinter eine maassgebende Persönlichkeit, die stark im Imprimatur für "veranlasste" Peptonarbeiten engagirt war, nicht undeutlich sehe.

In Prof. Hoppe-Seyler's Laboratorium war seit einer Reihe von Jahren eine Anzahl Schüler beauftragt gewesen, die Natur des Peptons, seine Zusammensetzung und sein Verhältniss zum ursprünglichen Eiweiss zu untersuchen. Durch diese Versuche, die

1) Zeitschr. f. phyșiol. Chem. 3. 58-62. 
alle von einerlei Misserfolg begleitet waren, zieht sich der gleiche Gang der Peptondarstellung: Anwendung roher Magenextracte, Neutralisation mit Baryt, Ausfällen des Baryt mit Sohwefelsäure, des Chlorwasserstoffs mit Silberoxyd, und Analyse der meist aschereichen Alcoholfällungen. Irgend eine Methode einer Reindarstellung, ein Versuch für das Analysirte die chemisch einheitliche Natur zu beweisen, sind nirgends enthalten. Diese Experimente sind von Möhlenfeld ${ }^{1}$ ) und ron Kistiakowskiª zuerst beschrieben worden, und später sind sie noch einmal von $\mathrm{Kossel}^{3}$ ) ebenso wiederholt worden, obwohl ich im Jahre 18742 Jahre vor Kossel's Mittheilung gezeigt habe, dass das Pepton die Zusammensetzung des Eiweisses hat, und zwar durch die Methode der fractionirten Fällung, die für die analysirten Körper die chemische Einerleiheit garantirt. Aber dies wurde wie gesagt von Hr. Kossel ignorirt, ohne dass er besseres oder überhaupt nur irgend etwas anderes an die Stelle gesetzt hätte; er verbrennt eben zu, ohne sich um Reindarstellung allzu ängstlich zu quälen. Das war 1876.

Der Leser sieht, ich habe es mit dem Reclamiren nicht eilig gehabt, ich habe vielmehr lange dazu geschwiegen, und zwar nicht nur über die genannten Originalien selbst, sondern auch über die Darstellung, die Hoppe-Seyler in seinem Handb. d. phys. und path. chemischen Analyse (Berlin 1875 anf 248 und 249) tiber das Pepton zu geben nicht verschmähte. Ich habe vielmehr die Sache auf sich beruhen lassen, in der Ueberzengung die Qualität der Arbeiten werde für sich selbst sprechen, und die Beurtheilung beim objectiven und sachverständigen Leser keine Verwechslungen veranlassen. Das ist nun wohl auch zugetroffen und ich kann als Gewährsmänner, die Jeder gern anerkennen wird, Go rup-B esa nez und $K$ ühne anfïhren. Gor u p-Besanez ${ }^{4}$ ) sagt in seiner milden und ruhigen Kritik: „diesen positiven Ergebnissen gegenüber", es sind die Arbeiten gemeint, die das Pepton ebenso zusammengesetzt wie das Eiweiss finden, also die von Thiry, mir und Herth, „verlieren gegentheilige Angaben von Möhlenfeld und Kistiakowski, nach denen die Peptone bis zu $10 \%$ C. weniger enthalten würden, wie

1) Pflügers Archiv 5. 381.

2) Daselbst 9. 438.

3) Daselbst 13. 309.

4) Lehrbuch d. physiol. Chem. 4. Aufl. p. 147. 
die Eiweisskörper, ihren Werth um so mehr, als ihre Methoden der Darstellung der Peptone die abweichenden Resultate unzweifelhaft beeinflusst hatten." In gleichem Sinne kann ich auch Kühne eitiren ${ }^{1}$, und das passt mir hier gerade in sofern recht gut, als ich mich punkto der Darstellung meiner Versuchsergebnisse in den Werken von Hoppe-Seyler zu letzterem in einer ähnlichen Lage befinde, als sich Kühne zu Hoppe-Seyler befand. Auch meine Arbeit wird von H.-S. als ihm nicht passend, bei Seite liegen gelassen, denn einige Laboratoriumspractikanten, die so rasch bei $\mathrm{ihm} \mathrm{zu}$ Schriftstellern avanciren, haben etwas anderes herausgebracht, und die werden gehalten auf Kosten jeden Restes nüchterner Darstellung. Kühne kommt in seiner Erwiderung an Hoppe-Seyler gelegentlich auch auf die Gleichheit der Zusammensetzuug von Eiweiss und Pepton, was wie er sagt ,neuerdings wieder von Maly gegen Hoppe-S eyler und Möhlenfeld bestätigt worden und von H.-S. als richtig zugegeben zu sein scheint".

Darin lässt also $\mathrm{K} \ddot{u} \mathrm{~h} n$ e gleichfalls ganz deutlich erkennen, dass für ihn diese Zusammensetzung und unzerstiuckelte Eiweissnatur des Peptons eine fertige Sache ist. Aus den Citaten dieser beiden bedeutenden Fachmänner, denen ich, was aber überflüssig ist, noch Andere anfiigen könnte, ist zu entnehmen, dass ich recht hatte vorauszusetzen, der Stand der Frage werde sich ohne weiteres erkennen lassen. Nur von einer Seite ist das nicht geschehen, denn während Kühne und ich endlich auch von Hoppe-Seyler's Seite stillschweigend - und das hätte ja gentigt - eine Zustimmung glaubten annehmen zu dürfen, zeigt sich, dass die Vermuthung irrig war, dass H. S. noch immer sich nicht beruhigt, dem Pepton das zu geben was ihm gebührt, denn er sendet nochmals den schiffbrïchigen Herrn Kossel hinaus. Dass dieser allerneueste, freilich etwas zahme Angriff, der mir eben den erwähnten Anlass gab, mich endlich dagegen zu wehren, wesentlich H.-S.'s Werk ist, entnehme ich daraus, dass er in dessen Laboratorium ausgeführt ist, von Jemanden, der für sich allein noch keine Gewähr zu leisten vermag.

Dies der Grund, der mich veranlasst, ausführlicher auf die Entwicklung der Peptonlehre einzugehen, die Verwirrungen zu beseitigen und meinen Antheil, der vorläufig grösser ist, als der

1) Untersuch. aus d. physiol. Institute Heidelberg. Band 2. Heft 1. p. 66. 
der ïbrigen Peptonbearbeiter zusammen, hervorzuheben. Würde es sich einfach um die Zusammensetzung eines der vielen tausend gleichgültigen Körper handeln, so lohnte sich mir eine so lange Erwiderung nicht; das Pepton aber nimmt im Thierchemismus, in der Verdaungs- und Ernährungslehre eine so hervorragende Stellung ein, wie ausser dem eigentlichen Eiweiss kein anderer Körper; der Nachweis seiner Isolirung als einheitlicher Körper, resp. der Beweis für die Existenz nur eines (Pepsin-) Peptons ist mühsamer gewesen als ein ähnlicher Nachweis in anderen Fällen; die Erkennung der Zusammensetzung des Peptons hat endlich den Schlüssel für die physiologische Bedeutung desselben geliefert, welche ohne die erstere ganz unvermittelt da stehen wïrde.

Ich werde daher zuerst zeigen, was vor meiner Peptonarbeit vorhanden war, werde beleuchten, wie die Herren Möhlenfeld und Consorten hantirt haben, was $\mathrm{ich}$ in die Untersuchung Neues eingeführt habe, was nach mir H. Kossel geleistet hat, und endlich unter einem, wie dies alles Hoppe-Seyler in seinem Werke darzustellen beliebt hat.

Der erste der in der Peptonchemie genannt wird, ist Lehmann; derselbe sagt in seinem Handbuch d. physiol. Chemie 1853 2. Aufl. I. 318, die Peptone (er spricht im Plural) seien weder im Kohlenstoff- noch Wasserstoff- noch Stickstoff- noch Schwefelgehalt von Eiweiss verschieden. Für diese Angaben fehlt aber eine Originalarbeit, es fehlen die analytischen Daten, nur für den $\mathrm{S}$ werden solche angefuhrt. Da der Schwefelgehalt aber am wenigsten massgebend ist, die Präparate auch eine Collection ron Aschebestandtheilen - kohlensaures Alkali, kohlensaurer Kalk, schwefelsaures Alkali - enthielten, Lehmann sonst nicht zögerte, analytische Angaben mitzutheilen, er das oben Gesagte aber nur nebenbei in sein Buch in Petit-Druck aufnahm, so kann er selbsit keinen besonderen Werth darauf gelegt haben. Endlich ist noch zu betonen, dass man zu Lehmann's Zeit noch kein Mittel kannte eine reinere Pepsinlösung herzustellen, sondern nur schleimhaltige Gemische anwenden musste, die Angabe also auf keinen Fall verlässlich sein konnte.

Schon nennenswerther sind die Analysen des Peptons von 
Thiry ${ }^{1}$, sie sind, wie der ganze Charakter der Arbeit zeigt an sorgfältig dargestellten Präparaten ausgeführt; allein, und das ist hier maassgebend, sie bezogen sich 1) überhaupt auf kein Verdau ungsproduct, d. h. sie waren nicht durch Pepsinwirkung hergestellt, sondern sie bezogen sich nur auf Producte, die durch andauerndes Kochen von Eiweiss mit Wasser hergestellt waren. Durch sie hat Thiry einen schätzenswerthen Beitrag zur Eiweisschemie, aber nicht zur Verdaungslehre geliefert, und wenn wir heute auch vermuthen, dass Thiry wirklich Pepton von der Zusammensetzung des Pepsinpeptons unter Händen gehabt, so glauben wir dies eben erst jetzt, nachdem das letztere dargestellt und mit dem ersteren übereinstimmend befunden worden ist. Ferner bezogen sich 2) Thiry's Analysen nur auf sehr baryumreiche Verbindungen mit wechselndem Barytgehalt.

Wie wenig in der That seiner Zeit von diesen beiden Arbeiten gehalten murde, kann derjenige entnehmen, der die 12 Jahre nach Thiry's Mittheilung erschienene 3. Auflage der den momentanen Stand der Kenntnisse gewiss repräsentirenden physiolog. Chemie von Gorup-Besanez vergleicht; darin ist weder von den Analysen Lehmanns, noch von denen Thiry's die Rede. Sie konnten also wichtig nicht erschienen sein.

Jedenfalls war bis da (ich will anerkennender urtheilen als Gorup-Besanez) die Lehre auf halbem Wege, unfertig zwar, aber nicht verfahren. Letzteres zu Stande gebracht zu haben, gebührt den Aufsätzen von Möhlenfeld und Kistiakowsky. Des Ersteren Anfsatz ist betitelt „iib er die Peptone d es Fibrin s". Man höre und staune wie dieselben dargestellt wurden. Statt nach irgend einer Methode ein reineres (substanzarmes) Pepsinpräparat zu bereiten, wurde wegen "Zeitmangels" (pag. 382 1. e.) nur künstlicher Magensaft hergestellt, indem Schleimhaut von Schweinemagen mit 0,1 procentiger $\mathrm{H} \mathrm{Cl}$ während 10-16 Stunden digerirt, abfiltrirt und die Digestion mit der Säure noch $z$ weimal wiederholt wurde. Wer jemals eine Schweinsmagenmucosa mit Säure digerirt hat, weiss, welche Massen Schleim aus der bestgewaschenen lederartig dicken Haut hervorquellen, und dass in der Zeit von 3 mal 10 oder gar 3 mal 16 Stunden (denn es wurde die Digestion zweimal wiederholt (pag. 382) in der Regel bis auf wenige Mem-

1) Zeitschrift f. rat. Medic. 3 R. 1862. 14. 1878. 
branfetzen alles längst gelöst ist. Möhlenfeld hat nun die Infusa von 8 sage acht solcher Schweinemägen, die zusammen das riesige Quantum von 5000 C. C. betrugen, genommen, und mit dieser breiten Bettelsuppe sein Bischen Fibrin, nämlich $65 \mathrm{gr}$ trocknen Fibrins (oder $210 \mathrm{gr}$ feucht) verdaut! Er hat also mit seinem Pepsinreagens ein vielmal grösseres Quantum fester Substanzen unbekannter Art, worunter jedenfalls sehr viel Schleim und Leim, in den Versuch eingeführtil), als das ganze zu peptonisirende Fibrin betrug, und doch wird später angenommen, es stamme alles, was fest ist, nur vom Fibrin. Jeder Zoll Theophrastus Paracelsus!

Die weitere Behandlung geht davon aus, es sei nichts weiter als Pepton vorhanden; Möhlenfeld neutralisirt mit Barytwasser, kocht, dampft ein, beobachtet dabei selbst d en Geruch nach Leim, entfernt den Baryt mit Schwefelsäure, digerirt dann in der vermeintlichen Absicht den Chlorwasserstoff wegzubringen mit überschüssigem Silberoxyd (einer Substanz, die fast alles Organische oxydirt) und fällt endlich die lehmigtrübe vom Chlorsilber ablatufende, und alkalisch gewordene (in Folge der Einwirkung des Silberoxyds auf die Alkalichloride) Flïssigkeit mit Alkohol. Filtrat und Niederschlag sind silberhaltig, sie werden mit Schwefelwasserstoff gesondert behandelt. Ich will nur von dem Filtrat, worin die Hauptmasse organischer Substanz enthalten war, sprechen; sie war alkalisch, und musste nach der Behandlung mit Schwefelwasserstoff nun lösliche Sulfide enthalten; sie wurde eingedampft, wobei sie braungelb wurde, auch der Leimgeruch sich wieder einstellte, und endlich mit Alkohol gefällt. Der erhaltene äusserst zähe Niederschlag, Möhlenfeld's Pepton wurde analysirt; er gab C 47,65 H 8,36 N 15,55 , woraus Möhlenfeld die Formel C 143 H 301 N 40 so 62 allen Ernstes rechnet und auch mittheilt. Also eine Formel für aufgelösten Schweinemagen mit etwas Fibrinzuspeise! Selbst eine Molecularbestimmung belzommt

1) Ich wog jüngst den Fundustheil einer Magenschleimhaut eines jungen Schweins, er hatte $119 \mathrm{gr}$. Acht solcher Häute wiegen also 952 gr oder nahe zu ein Kilo. Nehmen wir an, es sei darin wie durchschnittlich in den Geweben $3 / 4$ Wasser und $1 / 4$ feste Substanz, so bekommen wir $238 \mathrm{gr}$ trockene Substanz und da sich nach so langem Digeriren in der Regel die ganze Haut verdaut, so waren 65 Fibrin mit 238 anderer Substanz verunreinigt! 
man noch davon zu lesen, indem mit Barytwasser im Ueberschuss versetzt, mit $\mathrm{CO}_{2}$ behandelt und das Filtrat trocken gedampft wurde. Daraus resultirte ein Aequivalent (soll heissen Molekuil) von 1000 , das dann aber wieder verdoppelt wird u. s. w.

Möhlenfeld's analytische Resultate, die er trotz „Zeitmangels" doch noch fertig brachte, sind nun in Hoppe-Seyler's Handb. d. phys. path. chem. Analyse 4. Aufl. p. 248 und in dessen Physiolog. Chem. II. Heft p. 227 übergega ngen; im ersteren Werk ist auch die angezogene Formel nebst zwei weiteren Formeln Möhlenfeld's für die in dem Silberniederschlage enthaltenen Peptone mitgetheilt; uberhaupt dominiren in dem ganzen Peptoncapitel Möhlenfeld's Angaben, was ich vorläufig bitte den Leser zur Kenntniss zu nehmen ${ }^{1}$ ).

Auf den Aufsatz von Kistiakowski, der nichts besser als die Leistung Möhlenfeld's ist, brauche ich - obwohl anch dies recht heiter wäre - nicht einzugehen, insoferne es sich dabei um die Pankreaspeptone handelt, und diese nicht streng hieher gehören.

So standen die Sachen, als ich 1874 meine Versuche über die Zusammensetzung und physiologische Bedeutung vom Pepton veröffentlichte ${ }^{2}$ ). Von folgenden aus der Arbeit deutlich ersichtlichen Voraussetzungen bin ich dabei ausgegangen: 1) es darf kein aufgelöster Magen als Verdauungsreagens, sondern es muss eine Pep-

1) Dabei passirt Hoppe-Seyler folgendes. Pag. 248 liest man: „Durch den Magensaft werden die Eiweissstoffe in verschiedene Körper umgewandelt, deren Eigenschaften weit von denen der Eiweissstoffe abweichen, und die untereinander weder in den Reactionen noch in der Zusammensetzung übereinstimmen." Auf der nächsten Seite 249 werden die Körper Möhlenfeld's besprochen, nämlich der (s. o.) im Filtrat enthaltene, die Hauptmasse ausmachende und der im Silberniederschlage befindliche. Von diesem letzteren sagt jetzt H.-S.: "und es gibt keine Reaction, die ihn von dem zuerst beschriebenen (im Alcohol gebliebene M.) Verdau. ungsproduct scharf unterscheiden lasse."

2) Pflüger's Archiv 9. 585. - Ich hatte damals keine Aufforderung Möhlenfeld's Arbeit so zu analysiren wie heute; dass ich sie aber auch damals völlig durchsah, ist deutlich im Passus p. 589 enthalten: „von früheren Arbeiten sind nur zu nennen die von Lehmainn, von Thiry und allenfalls die Versuche Möhlenfeld's." Und später p. 592 nenne ich Möhlenfeld's Präparate "künstliche Gemische." 
sinlösung genommen werden, die möglichst arm an fester Substanz ist. 2) Es mïssen die für die Analyse störenden Aschebestandtheile möglichst vollständig und ohne Eingriff starker Chemikalien (wie Baryt, Silberoxyd etc.) e ntfernt werden; 3) es muss ein Mittel gefunden werden, durch welches man die chemischeIndividualität des analysirtenPeptons sichert. Diese Vorausbedingungen sind auf folgende Weise gelöst worden.

1) Die Pepsinlösung wurde nach einem combinirten Reinigungsverfahren (Brück.e und Krasilnikoff) dargestellt, und war, obwohl sehr wirksam, so arm an festen Bestandtheilen, dass - pag. 593 meiner Abhandlung - beim Abdampfen von 3 C.C. nur ein Rückstand von 1,5 mgr hinterblieb $=0,05 \%$. Es wurden also keine fremden Stoffe in nennenswerther Menge eingeführt, vollends keine aufgelösten Schweinemägen.

2) Ich habe gefunden, dass die Diffusibilität der Peptone resp. des Peptons kleiner ist, als die, der durch die Neutralisation der salzsauren Verdauungslösung entstandenen Chloride, und letztere daher durch das nicht zu beanstandendeMittel der Diffusion zu Wasser fortgeschafft werden können. So erhielt ich Präparate mit einem bisher nicht erreichten geringen Aschegehalt, herab bis zu $0,5 \%$.

3) Da beim Pepton die ïblichen Mittel der Reinigung durch Krystallisation oder Destillation nicht anwendbar sind, die Bindung an Basen ebenfalls werthlos ist, so blieb zur Erkennung, ob das verdaute Fibrin wesentlich aus e iner Substanz besteht, od e r ob es ein Gemenge von mehreren verschieden zusammengesetzten Körpern ist, nur der Weg der fractionirten Fällung, das ist hier der einzig mögliche und darauf lege ich einen grossen Werth. Da diese ebenfalls durch nicht eingreifende Reagentien bewirkt werden musste, nahm ich starken Alkohol und fällte die mit reinstem Pepsin erhaltene durch Dialyse aschefrei gemachte Peptonlösung damit in 3 und in einem andern Falle in 4 Fractionen. Wer den Werth der gebrochenen Behandlung kennt, wird zugeben müssen, dass, wenn ein Gemisch von Substanzen vorliegt, die einzelnen Fractionen nicht untereinander gleich zusammengesetzt sein werden, dass, wenn sie aber gleich zusammengesetzt sind, dies vorläufig noch immer beweisst, es liege nur ein Körper vor, der daher von keinem zweiten verunreinigt sein kann. Daranf beruhte meine Art etwas ïber das Pepton zu er- 
fahren, und ich bin noch von Niemanden belehrt worden, dass sich mit den heutzutage bekannten Methoden der Untersuchung eine für den vorliegenden Fall bessere und iiberzeugendere Reindarstellung ausfïhren lasse.

Die Resultate sind die bekannten: 1) Die einzelnen Fractionen sind gleichzusammengesetzt, es gibt also nurein Hauptproduct der Verdaung, nur ein Pepton; 2) das Pepton unterscheidet sich in seiner Elementarzusammensetzung $\mathrm{nur}$ se hr wenig von der Muttersubstanz, dem Fibrin; die dafür gefundenen Zahlen $\mathrm{C} 51,4, \mathrm{H} 6,95$ und $\mathrm{N}$ 17,12\% fallen noch in die für Eiweisskörper überhaupt gefundenen Zahlen. 3) Das Pepton kann daher kein Spaltungsproduct vom Eiweiss sein. Ein Jahr darauf erschien Hoppe-S eyler's Werk, die phys. path. chem. Analyse, dessen Seiten 248 und 249 ich schon frïher angezogen habe. Nachdem darin wie erwähnt die Analysen Möhlenfeld's ausführlich mitgetheilt worden sind, wird meiner Untersuchung in nur 1//2 Zeilen in folgender Art gedacht: "Maly findet die nicht weiter gereinigten Peptone von der Zusammensetzung der Eiweisskörper," und dabei das richtige Citat aus Pflüger's Archiv, als Beweis dass die volle Arbeit vorgelegen hat. Hoppe-Seyler ignorirt also das ganze Beweisende and schiebt kurz mit einem dictatorischen „nicht gereinigt" die Arbeit bei Seite und anerkennt nur die classischen Leistungen Möhlenfeld's. In einem anderen später erschienenen Werke (Physiolog. Chemie p. 226) wird zwar mit meinen Peptonresultaten nicht glimpflicher verfahren, aber obwohl ich seither nicht mit einem Worte darauf zurückgekommen bin, nennt H.-S. doch jetzt meine Präparate "durch Dialyse gereinigt." Also einmal sind sie nicht gereinigt, dann sind sie durch Dialyse gereinigt. Der fractionirten Darstellung, auf die es am meisten ankommt, wird aber auch hier wie in •dem ersten der citirten Werke mit keinem Worte gedacht, die Hauptsache damit verschwiegen, und dadurch der relative Werth meiner Arbeit gegenuber den von H.-S. begünstigten und gegenuiber allen frïheren Arbeiten überhaupt bis zur Unkenntlichkeit entstellt.

Es steht gewiss Hoppe-Seyler frei, nur das zu glauben und in seinem Laboratorium zu lehren, was man dort gefunden hat oder gefunden zu haben vermeint, und das was dagegen ist, seinen 
Schïlern zu verschweigen, aber ich muss mich dagegen verwahren, dass eine gleiche Behandlung in Werken Platz greife, die bestimmt sind und beflissen scheinen, den Stand der Kenntnisse objectiv darzulegen.

Etwa 2 Jahre später 1876 wird Herr Kossel von HoppeSeyler angeregt ${ }^{1}$ ), sich mit dem Gegenstande zu beschäftigen, und der Anlass zu neuer Untersuchung von Herrn Kossel darin gefunden, dass er sagt, „entweder ist bei dem Möhlenfeld'schen Verfahren dureh das Silberoxyd eine Oxydation oder Hydratation erfolgt, oder die Präparate Maly's waren nicht hinreichend gereinigt". Mit diesem scharfen Calciul, dessen erste Vermuthung von mir (Pflüger 9,592) ansgesprochen wurde, geht Herr Kos sel an die Arbeit. Auf die Idee, dass etwa.Möhlenfeld's Peptone nicht himreichend gereinigt gewesen wären, kommt er gar nicht zu denken als den Traditionen des Laboratoriums zuwider. Kossel stellt also nach der alten Schablone wie Möhlenfeld Pepton dar, das er aber gegenïber letzterem insoferne $m$ in der verunreinig $t$, als seine Verdaungsbrïhe nicht von 8 , sondern, wie aus der Beschreibung hervorgeht, von nur einer Schweinsmagenmucọsa dargestellt wird. Nachdem mit Baryt neutralisirt, mit Kohlensäure behandelt und mit Alkohol ein barythaltiges Pepton gefällt war, sollte entschieden werden, ob das Digeriren mit Silberoxyd eine Aenderung in der Zusammensetzung hervorbringe oder nicht. Es wurde nach Ausfällung des Baryts in zwei Theile getheilt, der eine mit Silberoxyd behandelt, der andere nicht. Aber nicht einmal das beabsichtigte Resultatchen konnte Hr. Kossel zu Stande bringen, denn er hätte dann mit Ausnahme des Digerirens mit Silberoxyd, beide Portionen ganz gleich behandeln müssen; er hat aber die eine noch mit kohlensaurem Kalk digerirt, die andere nicht, also einen Vergleich selbst unmöglich gemach t.

Der weitere Theil der Arbeit Kossel's bietet des Interessanten noch mehr. Die eben erwähnte zweite Portion der nicht mit Silberoxyd behandelten barytfreien Peptonlösung wird mit kohlensaurem Kalk digerirt, eingedampft, mit Alkohol gefällt und zur Analyse hergerichtet. Die Substanz war natürlich in hohem Grade aschehaltig, sie hatte die riesige Menge von $13,48 \% \mathrm{Ge}$ -

1) Pflüger's Archiv 13. 309. 
sammtasche, darunter Chlor, Calcium und Schwefelsäure, und da die Einzelbestimmungen davon zusammen nur 12,4\% ergaben, ohne Zweifel auch etwas Alkalien. Ein solches Gemisch bringt ein sorgfältiger Analytiker nicht gerne in's Verbrennungsrohr 1), da verlässliche Resultate davon nicht erhalten werden können. Aber Herr Kossel analysirt doch und bekommt schliesslich im Mittel von 3 aber untereinander wie zu erwarten, schlecht stimmenden Analysen für die gesammte (aschenhaltige) Substanz: C 45,13; H 6,23, N 13,96 S 1,08; $\mathrm{Cl} 2,34 ; \mathrm{Ca} 5,68$ und 025,57 .

Obwohl nach dem Gesagten so erhaltenen Zahlen Niemand Verlässlichkeit wird zuschreiben können, so will ich doch das nicht dazu gehörige eliminiren, also eine kleine Rechnung machen, die darin besteht, dass ich die ganze Collection der Aschenbestandtheile abziehe und für den bleibenden organischen Rest von 87,6 den Gehalt an $\mathrm{C}, \mathrm{H}$ und $\mathrm{N}$ procentisch rechne. Dann bekommt man für die analysirte organische Substanz:

$$
\begin{aligned}
& \text { C } 51,51 \% \\
& \text { H } 7,13 \% \\
& \text { N } 15,95 \%
\end{aligned}
$$

Macht man also diese einfache und selbstverständliche Rechnung, so sieht man, dass selbst Kossel's schmutzige Hantirung mit dem Mageninfus und seine ungenauen Analysen nicht schlecht genug waren, die Zusammensetzung des Peptons völlig zu verläugnen, denn die vorstehenden Zahlen stimmen wenigstens in $\mathrm{C}$ und $\mathrm{H} \mathrm{zu}$ meinen, und fallen im Ganzen auch noch in die Grenzen der Eiweisszahlen.

Das zu finden, war Herrn $\mathrm{K} 0$ ssel versagt; er schweift in die Ferne, sein Schutzengel sendet ihm im Traum die Alanine und er schreibt: „obige Analysen ${ }^{2}$ ) zeigen (wodurch? M.) dass die Aschebestandtheile des Peptons in chemischer Verbindung mit demselben sind. Die Aschebestandtheile sind daher nicht von dem Gewicht der analysirten Substanz abzuziehen, sondern es ist bei der Berechnung der Analysen für sie eine entsprechende Gewichtsmenge des vertretenen Atoms oder der vertretene n A tomgruppe (welehe Gruppe? M.) einzusetzen." Weiter: „eine Ab-

1) Bei mir wäre eine solche Substanz dem Laboratoriumsbesen zur weiteren Behandlung übermittelt worden.

2) D. h. seine ursprünglichen, nicht die von mir umgerechneten. 
scheidung dieser Aschebestandtheile gelingt deshalb so schwer (gelingt ganz gut M.) weil das Pepton sowohl die Eigensehaften einer Säure als einer Base hat."

Díeser Rede Sinn ist dunkel, die Absicht nicht; Herr K os sel sagt, das Pepton ist eine Base, und deshalb muss es Säure binden; die Sach e i s t a ber di e, dass er die Säure (natürlich die Salzsäure) daran gebunden haben möehte, um die kleinen Kohlenstoffzahlen zu finden, die er finden will, und darum lässt er es in Geschwindigkeit eine Base sein, und damit es seine basischen Aschenbestandtheile binde, muss es auch eine Säure sein. Nun speculirt Herr Kos e l über drei Fälle; entweder sei sein Körper 1) Peptoncalcium in Verbindung mit Chlorwasserstoff, oder 2) es sei Peptoncalcium in Verbindung mit Chlorcalcium, oder 3) es sei Pepton, worin Wasserstoff durch Chlorcalcium ersetzt ist; er rechnet dann nach diesen 3 Theorien die Zahlen, findet sie für jede Berechnung anders (niedriger als die welche nach dem Ascheabzug resultiren), und sagt dann befriedigt: ,jedenfalls geht aus diesen Zahlen hervor, dass im Verdanungsprocess eine Aenderung in der Zusammensetzung des Eiweissmolekïls erfolgt ist, stärker als man nach Angaben Maly's erwarten durfte, eine Aenderung, die wohl nur auf dem Eintritt von Wasser in das Ḿlekül beruhen kann etc." Ko ssel constatirt auch, dass die alten Zahlen $M \ddot{o b l e n f e l d s ~ u n d ~} \mathrm{~K}$ istiakowski's von den seinigen differiren and quält sich fruchtlos $a b$, in einem entweder - oder diese Ursache zu errathen. Hier ist davon nur desshalb Erwähnung zu thun, um zu erinnern, dass die sämmtlichen unter Hoppe-Seyler's Führung zu Stande gebrachten Peptone nur darin übereinstimmen, dass jedes eine andere Zusammensetzung zeigte, und dass jedesmal andere Deutungen aufmarschiren, um das Misslingen zu bemänteln, ein im $\mathrm{H}_{0}$ ppe-Seyler'schen Sinne zusammengesetztes Product von constanter Beschaffenheit zu erhalten, d. b. ein solches das zum Eiweiss in keinem verständlichen Verhältnisse steht!

Kossel's Präparat, das trotz aller Misshandlung sich noch annähernd als Pepton verräth, indem es ähnlich dem Eiweiss zusammengesetzt ist, wird desshalb bis zur Unbrauchbarkeit verrechnet um zn erreichen, quod erat demonstrandam.

Ich will noch beleuchten, wie sehr sich Herr Kossel auf den Leim führen liess, mit obiger Berechnung, und zwar unter der von 
Kossel gemachten - aber völlig aus der Luft gegriffenen - Behauptung, das Pepton sei eine Base und Säure, es verbinde sich daher mit beiden gleichzeitig wieder.

Der eine Grund der dafür angegeben wird ist der, dass der Körper mit Alkohol gefällt war und doch Chlorcalcium enth i elt; das beweist aber nur, dass vom Pepton bei der Ausfällung Salze auf irgend eine Weise mit niedergerissen werden, es braucht das Chlorcalcium desshalb nicht verbunden zu sein. Ich habe chlorcalciumhaltiges Pepton vom Chlorcaleium durch die Dialyse so vollständig befreit, dass es complet aschefrei war. Der Grund des einfachen Vorhandenseins ist also für die chemische Verbindung irrelevant. Weiter wird geltend gemacht, und darauf legen $\mathrm{Kossel}$ in seiner Abhandlung und selbst Hoppe-Seyler (Physiol. Chemie Heft II p. 226) vielen Werth: das in der Peptonverbindung gefundene Chlor $(2,34 \%)$ und das gefundene Calcium $(5,68 \%)$ seien nicht in dem Verhältnisse wie im Chlorcalcium ${ }^{1}$ ), können also nicht als solehes im Peptonpräparat enthalten gewesen sein. Das ist richtig, aber sie können als basisches Chlorcalci um darin gewesen sein. Schon beim Abdampfen im Wasserbade wird eine Chlorcalciumlösung basisch! Dieser Grund beweist daher auch nichts.

Ich will aber weiter sogar das Unwahrscheinliche als wahrscheinlich und das Unbewiesene (dass eine molekulare Verbindung mit $\mathrm{Ca}$ und $\mathrm{Cl}$ vorgelegen habe) als bewiesen annehmen und sehen, wie sich unter solcher Annahme die Berechnung der ursprünglichen Analyse gestalten würde.

Wenn $\mathrm{Ca}$ und anderseits $\mathrm{Cl}$ chemisch an Pepton gebunden sind, kann das nicht anders sein als in den Alaninen (Amidosäuren) und Herr Kossel selbst deutet an, dass er auf diese denkt, denn er sagt in der noch zu besprechenden letzten Notiz ${ }^{2}$ ), das geschilderte Verhalten des Peptons bilde „eine werthvolle Analogie zu dem Verhalten der Amidosäuren."

Ich nehme also für diese Analogie ein einfaches Beispiel, z. B. Glyeocoll, einen Körper, der Herrn Kossel bekannt sein künnte. Derselbe verbindet sich, was für unseren Fall gut passt, mit salpeter-

1) Von jedem Element ist nur eine Bestimmung gemacht worden, dam her fraglich, ob sie überhaupt richtig waren.

2) Zeitschr. f. physiol. Chemie 3. 62. 
saurem Silber, oder mit Chlorkalium etc. Aber dabei wird kein Wasserstoff im Glycocoll ersetzt, noch weniger eine „A tomgruppe" wie das Kossel bei dem ,analog" sich verhaltenden Pepton annimmt, sondern es findet zwischen der Amidosäure und dem Salz einfache Anlagerung statt, nämlich:

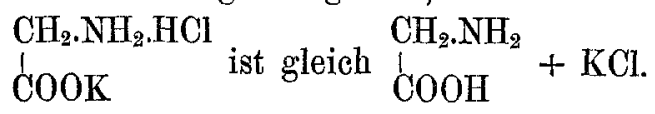

Daraus folgt, dass wenn im Pepton Mineralbestandtheile nach Art der Amidosäurengebunden sind, sie erst recht abgezogen werden müssen.

Darnach sind die Umrechnungen in Kossel's Abhandlung und Hoppe-Seyler's Handbuch zu beurtheilen, und die Spitze in des Ersteren Aḅandlung, die wesentlich gegen mich gerichtet war, kehrt sich um und thut ihre Pflicht.

Ich fahre in der Peptonchronik weiter; im Jahre 1877 hat R. Herth ${ }^{1}$ ) die Methode, die ich am Fibrinpepton ausgefüht habe, nämlich die fractionirte Fällung, am Hühnereiweisspepton durchgeführt. Das coagulirte Hühnereiweiss wurde durch Auslangen mit verd. Phosphorsäure fast aschefréi gemacht, die Pepsinlösung war dialysirt und die Verdauung dauerte kurz. Um keine Aschebestandtheile überhaupt hereinzubekommen, wandte Herth ein Verfahren an, das einen erfreulichen Fortschritt in der Peptondarstellung bildet; er verdaute mit Phosphorsäure und entfernte diese nach der Verdauung mit gefälltem Bleicarbonat wieder. Die Peptonlösung wurde mit Alkohol in 4 Fractionen gefällt. Dabei zeigte sich noch vollkommener als an den Fibrinfractionen, dass das Eiweisspepton gleich oder nahe gleich dem ursprïglichen Eiweiss zusammengesetzt ist, und das die Fractionen untereinander gleich sind. Im Mittel aller ergab sich C 52,33, H 7,05, N 16,72 für Pepton, während W u rtz im coag. Hühnereiweiss fand $\mathrm{C} 52,9 ; \mathrm{H} \mathrm{7,1;N} 15,6$.

Dasselbe fand Adamkiewiez bei einigen Analysen, und obwohl er meine und Herth's Resultate noch nicht kannte, so spricht er doch in seinem schönen Werke: Natur und Nährwerth des Peptons Berlin 1877. 45 die Ueberzeugung aus, „dass im Eiweiss durch die Magenverdauung der Complex der Elemente nicht gestört wird."

1) Zeitschr. f. physiol. Chemie 1. 277. 
Endlich hat, ich muss sagen, zu allem Ueberflusse, in einer ausführlichen neuesten Arbeit Henninger ${ }^{1}$ ) in Paris nochmals das Pepton von Fibrin, Eiweiss und Casein sehr sorgfältig dargestellt und analysirt; auch er findet die einzelnen Peptonfractionen gleich zusammengesetzt und seine Zahlen sind mit denen von mir und von Herth fast identisch. Ich setzte nun zum Vergleich die Mittelzahlen nebeneinander.

\begin{tabular}{|c|c|c|c|c|c|}
\hline Fibrin. & \multicolumn{2}{|c|}{ Fibrinpepton. } & Eiweiss. & \multicolumn{2}{|c|}{ Eiweisspepton. } \\
\hline Maly. & $\begin{array}{c}\text { Maly } \\
\text { (Mittel). }\end{array}$ & $\begin{array}{l}\text { Henninger } \\
\text { (Mittel). }\end{array}$ & & $\begin{array}{l}\text { Herth } \\
\text { (Mittel). }\end{array}$ & $\begin{array}{l}\text { Henning er. } \\
\text { (Mittel). }\end{array}$ \\
\hline C5 & 51,40 & 51,43 & 52,9 & 52,33 & 52,28 \\
\hline & 6,95 & 7, & 7 & 7,05 & 7,05 \\
\hline N 17,34 & 17,13 & 16.66 & $\left.15,6^{2}\right)$ & 16,38 & 16,38 \\
\hline
\end{tabular}

Wir stehen also einerseits vor einer Reihe wohl zusammenstimmender Resultate, denen zumeist Präparate von systematischer Fractionirung zu Grunde liegen, und anderseits vor einer Reihe nicht mit ersteren und nicht untereinander zusammenstimmender Resultate, über deren zu Grunde liegende Präparate und Rechnungskunststïcke das vorhergehende den nöthigen Aufschluss gibt. Jeder Unparteiisehe wird, ich habe schon Beweise dafür mitgetheilt, die ganze Frage als abgeschlossen betrachten; $d . h$. wir wissen jetzt und seit mehreren Jahren.mit jener Bestimmtheit, die dureh analytische Methoden überhaupt zulässig ist, dass Fibrin und Eiweiss nur je ein einheitlich zusammengesetztes Pepton als Verdauungsproduct liefern, und dass dieses Pepton noch die Zusammensetzung eines Eiweisskörpers hat, resp. ein solcher ist. Ob dabei nun das Pepton mit dem Eiweiss völlig isomer ist oder ob dabei eine kleine auf ein Molekül Wasser zu beziehende Zusammensetzungsdifferenz sich ergibt, ist dabei für meine Behauptung vorläufig gleichgïltig, denn das ist bei einem so hoch zusammengesetzten Körper ïberhaupt durchs Analysiren nicht herauszubringen. Aber das lässt sich behaupten, dass Pepton und Eiweiss entweder gleich zusammengesetzt sind oder dass sie nur um wenig differiren, $k \mathrm{urz}$ das $\mathrm{s}$ die Peptone von ihren resp. Muttersubstanzen nicht mehr abweichen, als diese d. h. die andern, also eigentlichen Eiweisssubstanzen untereinander. Dieses wichtige zuerst von mir bestimmt gefundene, dann von meinem Schüler Dr. Herth

1) Jahresb. d. Thierchem. 8. 24. Aus Compt. rend.

2) Zu klein, weil mit Natronkalk bestimmt. 
330 Richard Maly: Ueber d. Verwirrungen u. Entstellungen i. d. Peptonlehre.

bestätigte Resultat findet endlich seinen Abschluss in den bekannten Ernährungsversuchen, nach welchen ein Thier durch Pepton ohne alles andere Eiweiss so ernährt werden kann, wie durch Eiweiss selbst. Diese Fragen stehen in innigstem Zusammenhange; welche Hypothesen wären nöthig; wenn man die Irrlehre Hop pe-Seyler's annehmen witrde: dass die Peptone in ihren Eigenschaften weit von denen der Eiweissstoffe und untereinander abweichen.

Und doch wird nochmals versucht gegen den Strom zu schwimmen; derselbe Herr $\mathrm{K}$ osse l, der so klug thäte, sich in der Peptonfrage recht ruhig zu verhalten, bringt aus Hoppe-Seyler's Laboratorium im Märzheft der Zeitschr. f. phys. Chemie 1. c. eine neue Notiz, deren thatsächlicher Inhalt zu nichtssagend ist, als dass ich davon Notiz zu nehmen hätte, die aber doch, wenngleich etwas nachgebend, noch sich auf den negirenden Standpunkt stellt. Kossel sagt jetzt nicht mehr ,entweder sind Maly's Peptone nicht hinreichend gereinigt etc.", sondern er befindet sich nur mehr in folgendem Dilemma: "die Differenzen zwischen beiden Werthen (den von mir und Henninger einerseits und den älteren von Kossel anderseits M.) können auf verschiedene Weise erklärt werden. Entweder gibt das freie Pepton bei $110^{\circ}$ Wasser ab, während die Verbindung des Peptons dieses Wasser bei jener Temperatur zurück behält, oder es enstehen bei der Pepsinverdauung anfangs Producte, welche die von Maly und Henninger gefundene Zusammensetzung haben, späte Producte mit niederem Kohlenstoffgehalte." Nachdem dann eine neue Analyse angeschlossen wird, die einen niederen Kohlenstoffgehalt ergibt, vermeint $K$. bewiesen zu haben, "dass die Zusammensetzung der Verdauungsproducte von der Stärke der Pepsinwirkung abhängt." Damit führt Herr Kossel eine ganz neue Hypothese ein. Wenn die Zusammensetzung der Verdaungsproducte von der Stärke der Pepsinlösung abhängt, so würde das so viel heissen, als Pepsinlösungen verschiedener Stärke wirken qualitativ verschieden, eine stärkere Fermentlösung erzengt etwas anderes, eine verdünntere erzeugt wieder etwas anderes!

Damit dürfte das Ultimatum von Gedankenlosigkeit erreicht worden sein.

Ich habe schliesslich noch mein Bedauern auszusprechen, dass ein Laboratoriumsvorstand von solchen Verdiensten wie 
Richard Maly: Abwehr in Angelegenheit des Hydrobilirubins(Urobilin). $\mathbf{3 3 1}$

Hoppe-S eyler jedem noch nicht flügge gewordenen Laboratoriumspraktikanten die Feder in die Hand gibt, und dadurch eine Literatur schafft, deren ephemeren Werth weiter hinaus stïtzen zu lassen, von Ihm selbst schon peinlich empfunden werden durfte.

\section{Abwehr in Angelegenheit des Hydrobilirubins (Urobilin).}

Von

\section{Richard Maly}

in Graz.

Unter den physiologisch-chemischen Schillerversuchen, deren Publication Hoppe-Seyler's Laboratorium fabriksmässig betreibt, sind in der jüngsten Zeit noch zwei Mittheilungen enthalten gewesen, die sich ziemlich ausschliesslich gegen mich richten, die aber durch ibre Leerheit an neuem Material zu den Schluissen nicht berechtigen, die dort gezogen worden sind. Es sind Kossel's Notiz über "die ehemischen Wirkungen der Diffusion"1) und Disqué's Mittheilung über „Urobilin“"2). Um nicht in gar zu ungebührlichem Umfange dieses Journal für Klarstellungen, die nicht viel neues bringen können, in Anspruch nehmen zu müssen, werde ich für diesmal nur noch der zweiten Mittheilung, Disqués Urobilin gedenken.

Die Lectïre dieser Untersuchung (s. v. v.) hat mich nicht nur desshalb interessirt, weil das kïnstlisch dargestellte Urobilin von mir herrithrt, sondern vor allem dadurch, dass am Ende derselben pag. 271 zehn Thesen in durchschossenen Lettern als Resultate der Untersuchung zusammgestellt worden sind, von denen Nr. 1 lautet: „Das von Maly kinstlich dargestellte Urobilin ist

1) Zeitschr. f. physiol. Chem. 3. 207-211.

2) Zeitschr. f. physiol. Chem. 2. 259-272. 